\title{
A Sustained Proximity Network for Multi-Mission Lunar Exploration
}

\author{
Jason A. Soloff' \\ Goddard Space Flight Center, Greenbelt, Maryland, 20771 \\ Gary Noreen ${ }^{\dagger}$ and Leslie Deutsch ${ }^{\dagger}$ \\ Jet Propulsion Laboratory, California Institute of Technology, Pasadena, California, 91109 \\ and \\ David Israel I $^{\S}$ \\ Goddard Space Flight Center, Greenbelt, Maryland, 20771
}

\begin{abstract}
The Vision for Space Exploration calls for an aggressive sequence of robotic missions beginning in $\mathbf{2 0 0 8}$ to prepare for a human return to the Moon by 2020 , with the goal of establishing a sustained human presence beyond low Earth orbit. A key enabler of exploration is reliable, available communication and navigation capabilities to support both human and robotic missions. An adaptable, sustainable communication and navigation architecture has been developed by Goddard Space Flight Center and the Jet Propulsion Laboratory to support human and robotic lunar exploration through the next two decades. A key component of the architecture is scalable deployment, with the infrastructure evolving as needs emerge, allowing NASA and its partner agencies to deploy an interoperable communication and navigation system in an evolutionary way, enabling cost effective, highly adaptable systems throughout the lunar exploration program.
\end{abstract}

\section{Introduction}

$\mathrm{T}$

HE Vision for Space Exploration ${ }^{1}$ begins with a campaign of robotic precursor missions to the Moon. In response to this call, NASA created the Robotic Lunar Exploration Program (RLEP). RLEP consists of a multimission sequence of reconnaissance orbiters, landed elements and technology demonstrations whose combined mission is to characterize the lunar environment, prepare for a safe, assured human return, and validate the technology and operations concepts necessary for a sustained human presence beyond low Earth orbit.

This paper describes a strawman network architecture to enable multi-mission operations and proximity crosssupport in the lunar environment. The concept builds on the lessons learned and successes of JPL's Mars Network by integrating proximity relay operations, interoperable protocols, and autonomous communications into the RLEP mission set. By designing individual RLEP missions with proximity networking in mind, a highly capable, available and reliable in-situ communication network can be built as the program evolves.

NASA envisions launching robotic missions to the Moon on an approximately annual basis leading up to the human return to the Moon between 2015 and 2020. International space agencies have also identified aggressive robotic lunar exploration campaigns. The significant number of missions to the Moon over the next decade creates a unique "critical mass" that could be leveraged into an evolvable, sustainable lunar communications network, with each new mission adding new capabilities, extending and replenishing the existing network.

This paper reviews lunar exploration requirements (both known and assumed) and the potential mission set that has been proposed to meet them. It then considers the communication and navigation requirements necessary to

\footnotetext{
- Communication Systems Engineer, Microwave and Communication Systems Branch, Code 567, Member.

+ Senior Engineer, Mission and Systems Architecture Section, M/S 301-170S, Senior Member.

* Manager, Architecture and Strategic Planning Office, Interplanetary Networks Directorate, MS 303-401, Member.

${ }^{\S}$ Leader, Advanced Technology Development Group, Microwave and Communication Systems Branch, Code 567.
}

1

American Institute of Aeronautics and Astronautics 
support these missions. Finally, an evolving proximity network implementation is described, discussing how the concept can extend and adapt to provide a continuous, sustained mission cross-support capability.

\section{The Robotic Lunar Exploration Program}

To meet the first objective of the Vision for Space Exploration, NASA created the Robotic Lunar Exploration Program, the objectives of which are to perform science and measurements vital to lunar exploration, demonstrate key technologies and operations concepts, and deploy initial infrastructure to enable a sustained human presence at the Moon and beyond. RLEP is managed by the Goddard Space Flight Center and consists of a series of individual missions, the first of which is the Lunar Reconnaissance Orbiter (LRO) scheduled to launch in 2008. LRO will be followed by surface landed elements and additional reconnaissance missions to improve our knowledge of the Moon and the Cis-Lunar environment. Follow-on RLEP missions are expected to prove technologies critical to the success of the Crew Exploration Vehicle and other Constellation systems, as well as pre-deploy infrastructure to support extended duration human exploration of the Moon.

The early science objectives of RLEP are derived from the NASA Decadal Study and from the input of the planetary science and space operations communities and include lunar topography, radiation environment, regolith composition, resource identification, and gravity mapping. The objectives of each RLEP mission are derived from the questions asked and answers learned during previous missions. If a mission detects from orbit the likely presence of resources, a later mission may be targeted to land at that site and directly sample the surface to provide "ground truth" measurements confirming the earlier discovery. In this way the program is responsive to new discoveries, new objectives, and emerging requirements of NASA's exploration and science programs.

LRO addresses the most pressing of these investigations. It is a multi-faceted mission, whose measurement set provides data products vital to exploration planning while filling large gaps in our scientific knowledge of the Moon. The measurements from LRO's selected instrument set consist of high resolution imagery and topographical measurement, radiation characterization, and resource identification. The mission will operate in a nominal $50 \mathrm{~km}$ lunar polar orbit in order to provide high fidelity science. The spacecraft will collect and retum over $450 \mathrm{~Gb} /$ day of science data, delivering over 164 terabits during its one year primary mission. This data volume is unprecedented for a non-Earth science mission. Following LRO's primary mission, the mission will enter a planned "extended mission" phase during which the spacecraft will be placed in a low maintenance orbit where it will perform additional science and likely function as an infrastructure resource for later missions. It is LRO's extended mission, and the extended missions of other lunar orbiting spacecraft, that provides the opportunity to leverage a local lunar proximity network.

The second RLEP mission, to launch one year following LRO, is envisioned to consist of one or more landed elements to be deployed to the lunar surface from an orbiting host carrier. As previously mentioned these landers will target likely resource concentrations and provide in-situ measurements to confirm or disprove LRO's findings. Multipurpose probes may also verify the lunar radiation environment and perform other surface science. Later missions will provide additional measurements in this manner, and eventually verify exploration technologies including power generation, and in-situ resource utilization.

Missions throughout the RLEP sequence will rely on high volume data retrieval and precision navigation. Landers and roving elements must be targeted to specific sites to confirm measurements made from orbit. Eventual pre-deployed infrastructure must be delivered reliably to specific locations for later use by follow-on robotic and human missions.

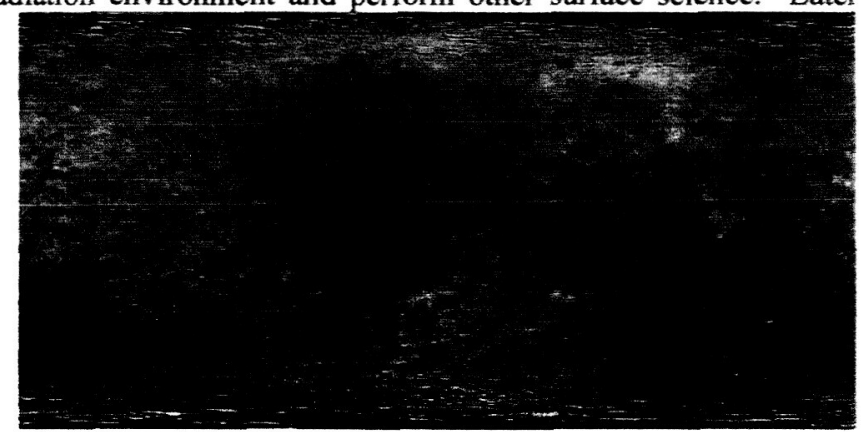

Figure 1 - Lunar Prospector Hydrogen (water) Map Image from the National Space Science Data Center

\section{RLEP Use of Cross-Support}

The Moon's topography, coupled with its tidally locked orbit about the Earth, creates unique locations near the poles that are in permanent shadow. Lunar scientists believe that these permanently shadowed regions are likely to contain trapped volatiles frozen into the regolith. Measurements performed by the Lunar Prospector and Clementine missions $s^{2,3}$ indicated the presence of resources including water ice in several craters near the lunar south pole ${ }^{4}$ (Figure 1). These resources may exist in significant quantities and provide a convenient resource for future human missions. 
The topography that protects these sites from solar illumination also hides them from view of the Earth. In order to make in-situ measurements in permanently shadowed regions, it is necessary to find a non-line-of-sight method for communication between Earth and the landed surface probe. Proximity relay, in which one spacecraft serves as a communication intermediary, provides an elegant solution to this problem.

In proximity relay operations, one venicle, possibly masked by terrain as revealed at the Moon's south pole by the Clementine ${ }^{2}$ mission (Figure 2), and therefore out of view of its intended recipient, transmits its data to a separate vehicle that is in view. A likely scenario is a landed probe communicating with an orbiting carrier spacecraft.

The receiving vehicle records and stores the data and later transmits it, along with its own data, to Earth or another intended destination. Transmission of commands to the masked vehicle is accomplished by initially transmitting them to the relay, which then delivers the commands to the intended user during a later line-of-sight contact period.

Proximity relay operations, in which one mission provides cross-support to another, may provide the only realistic method for communication with probes deployed to permanently shadowed regions of the Moon.

Navigation will also play a key role in successful lunar robotic and human exploration. Initial robotic

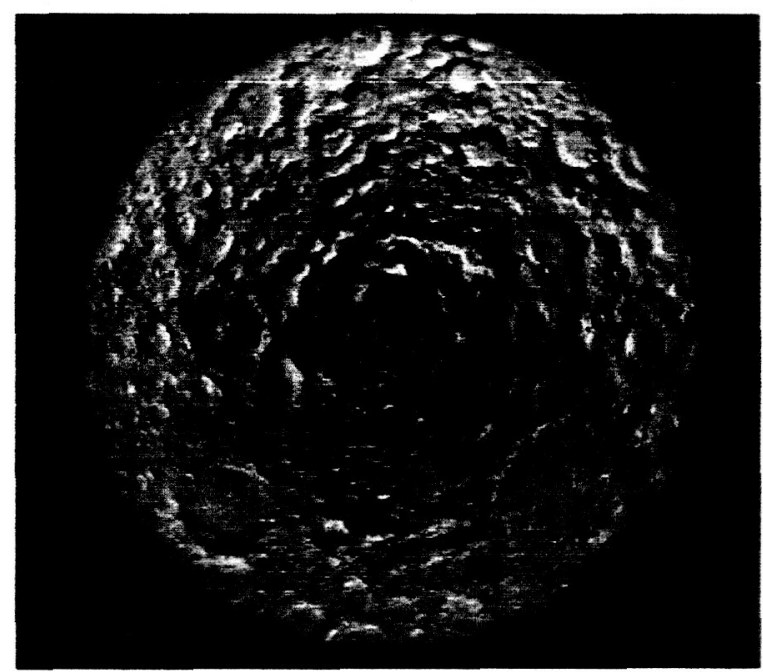

Figure 2 - South Pole Lunar Surface

Image from the National Space Science Data Center missions will need to determine their location to identify where measurements are made, allow for accurate maneuvering, and provide for the targeting and terminal guidance of landed elements such as probes and rovers. The GPS constellation which provides this capability at Earth is not able to support lunar operations. Traditional direct-from-Earth Doppler and range measurement employed by systems such as NASA's Deep Space Network (DSN) and Tracking and Data Relay Satellite System (TDRSS) can only provide navigation support to a single user per beam (with each beam requiring its own antenna asset) and cannot provide support to vehicles operating on the far side of the Moon. Proximity communications could provide navigation support to these missions without occupying scarce Earth-based ground assets, as well as provide capability on the far side.

As RLEP missions grow in complexity, evolving to demonstrate key exploration technologies, and as more missions are undertaken by NASA and its international partners, the number of assets (landed probes, rovers and orbiting spacecraft) operating in the lunar environment will increase. Individual mission and campaign goals will likely require these vehicles to communicate to exchange data, coordinate actions and provide navigation information.

The potential number of simultaneous vehicles, as in a distributed robotic surface explorer or an advanced in-situ resource utilization demonstration, may make direct-to-Earth communication impractical even with all mission assets in view due to the number of simultaneously supported communication links. In this case the most practical method of communication may be to establish a "local area network" consisting of the landed elements and one or more orbiting assets to act as a communication relay and router. Information moving between mission elements can then remain in the lunar environment (the local network) while measurement data, engineering telemetry and operational command and control can be coordinated and distributed through the relay. This approach limits the operational complexity and cost of providing Earth-to-Moon communications for the mission.

\section{Mars Network Concept for Mission Cross-Support}

NASA's Science Mission Directorate has established a policy by which any mission to Mars having an expected on-orbit lifetime exceeding one year must carry a proximity relay capable communication system. This policy has established near Mars an ad-hoc relay network able to support advanced mission concepts. An excellent example of this policy's success is the recent Mars Exploration Rover (MER) missions, Spirit and Opportunity, still collecting data on the Martian surface, whose communications were conducted primarily through pervious years' Mars orbiters.

3

American Institute of Aeronautics and Astronautics 
Mars orbiters with relay radios are enabling exciting in-situ missions to the Red Planet. Spirit and Opportunity have been tracked by NASA's Mars Global Surveyor (MGS) orbiter since their arrival at Mars and have relayed data to Earth through both Odyssey and MGS after landing on January 4th and 26th, respectively. Rovers have also communicated through the Mars Express spacecraft.

NASA added relay radios to Mars Global Surveyor and Mars Odyssey specifically to provide relay services to other missions. As such, the relay radios are part of a new way of exploring other planets: through a long term program, with each mission building on others to establish an interplanetary internet for the support of future missions.

NASA will add Mars Reconnaissance Orbiter (MRO) to the Mars Network in 2005. MRO will carry the first Electra $^{6}$, a frequency-agile software relay radio, and will have high rate X-band and Ka-band Direct-To-Earth links. While the Mars Network of science orbiters with relay radios has greatly improved our ability to navigate and communicate with Marscraft (landers, rovers, aerobots, and orbiters in the vicinity of Mars), its capabilities are limited because it has been constructed by adding relay radios to orbiters optimized for science missions rather than for communications.

NASA will augment the Mars Network in 2010 with the Mars Telecommunications Orbiter (MTO), the first interplanetary spacecraft optimized for relay communications services. Figure 3 depicts the Mars Network prior to the addition of MTO to the constellation.

MTO will be placed into a high orbit selected specifically for its relay mission. MTO will have the most advanced communications system ever put on an interplanetary spacecraft, with high performance X- and Ka-band links to Earth, high performance UHF and X-band relay links to other Marscraft, and an experimental laser communications payload for Direct-ToEarth (DTE) communications.

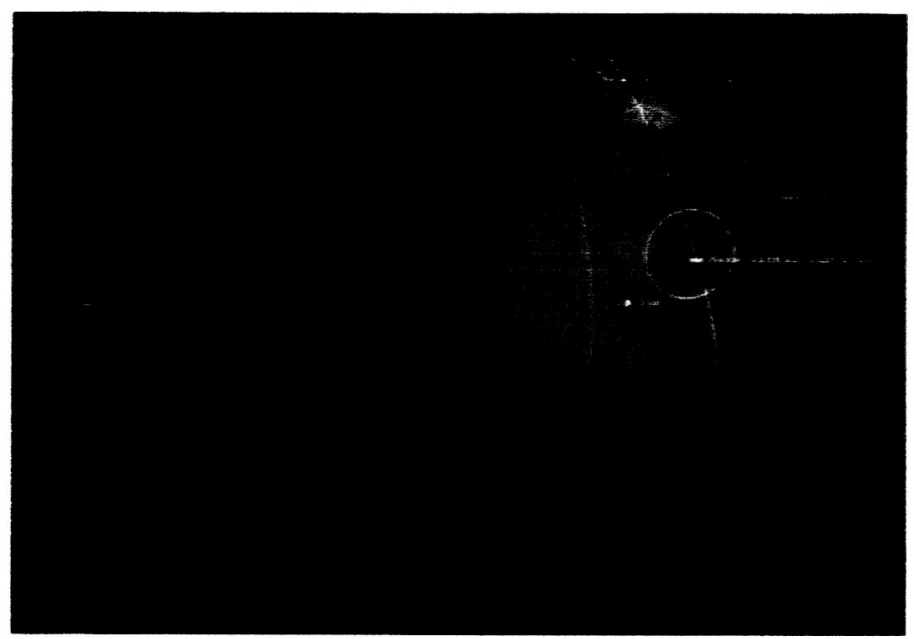

MTO will dramatically increase both the data return from other missions sent to Mars and the amount of time and frequency of contacts to Mars missions, Figure 3 - Mars Network Constellation fundamentally improving our ability to monitor and control Marscraft and leading to more flexible and reliable operations. The lessons learned at Mars with respect to operations and communication networking concepts can be directly applied to similar problems at the Moon and other destinations throughout the solar system.

\section{Evolution of the Mars Network}

The Mars Network consists of Mars orbiters with radios capable of relaying communications to and from other Marscraft and ground stations on Earth. The Mars Network currently consists of the NASA Mars Global Surveyor (MGS) and Mars Odyssey orbiters. The origin of the Mars Network can be traced to a French Mars balloon mission: Jacques Blamont of CNES proposed to send balloons to Mars on what eventually became the ill-fated Russian Mars '96 spacecraft. To communicate with these balloons - which would have had no DTE capability - CNES developed the Mars Balloon Relay (MBR). A CNES MBR was first sent to Mars on another ill-fated spacecraft in 1992: NASA's Mars Observer. A CNES MBR successfully reached Mars on MGS in 1997.

The international interest in relay communications at Mars led to the development of a space relay radio standard by the Consultative Committee on Space Data Standards (CCSDS): the Proximity-1 standard ${ }^{5}$. Proximity-1 is a flexible bidirectional protocol providing several operating modes and levels of service. While initially developed for Mars missions, it is intended to be used for other space missions as well. The CE 505 relay radio sent to Mars on the NASA Mars Odyssey orbiter in 2000 was the first to implement the CCSDS Proximity-1 protocol. This was followed by the MELACOM radio on Mars Express.

MGS, Odyssey and MRO have nadir-pointed UHF Low Gain Antennas for relay communications. The platforms on which each of these LGAs is placed are shared with several scientific instruments, resulting in irregular, less than optimal performance patterns. Mars Telecommunications Orbiter (MTO), to be launched in 2009, will have high 
performance steered X-band and UHF relay antennas on a dedicated relay antenna platform. Because these antennas are steered, they will normally operate near their peak gain - unlike the UHF relay antennas on other Mars orbiters.

The Mars Network evolved from an experiment, but has become a cornerstone of NASA's deep space exploration concepts. The phased approach to building the network, with each new orbiting mission augmenting the network's deployed capability has provided significant benefits for a modest additional investment in each mission. Figure 4 shows the deployment phasing of the existing and near-term Mars Network.

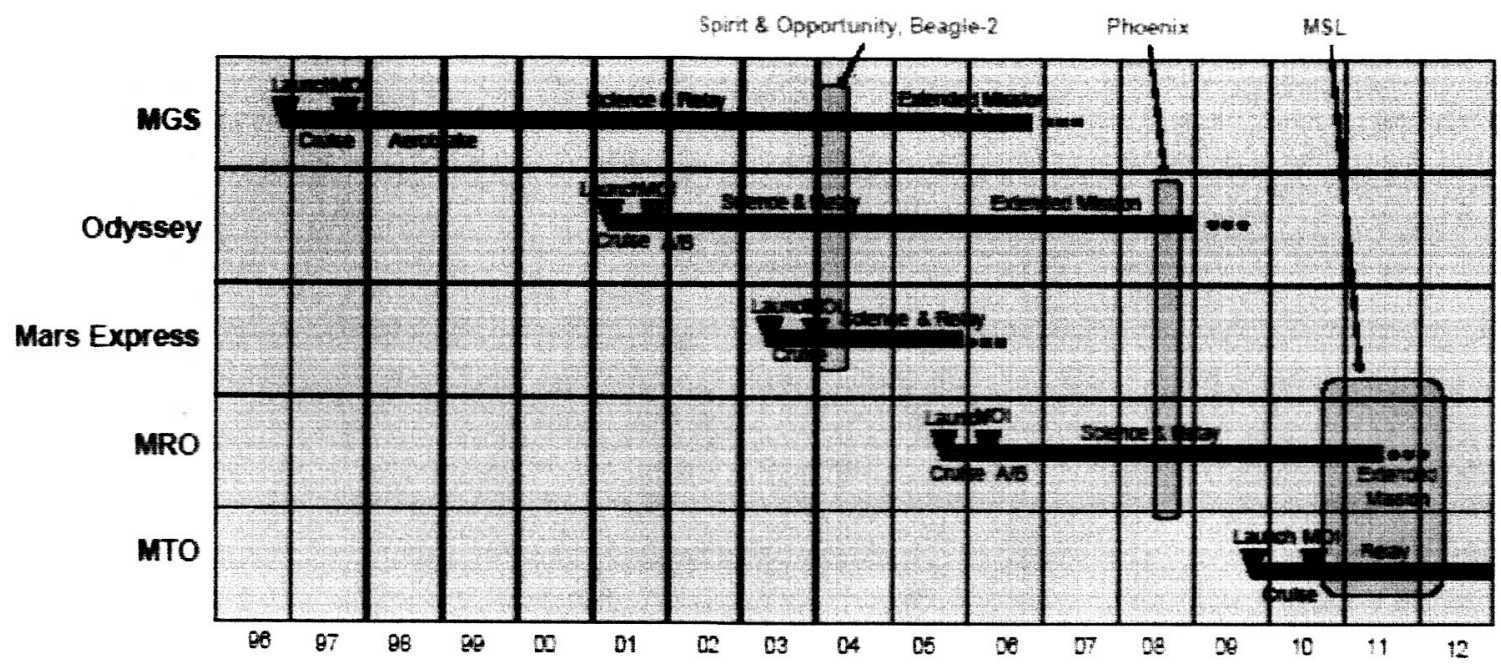

Figure 4 - Deployment Phasing of the Mars Network

\section{Lunar Proximity Network Architecture}

As demonstrated through the Mars Network concept, ensuring interoperability between multiple missions requires both technological capability and standardization. JPL has demonstrated initial success by standardizing on the CCSDS Proximity-1 protocol for relay applications on their deep space missions, and through the use of basic software radio hardware in the form of the Electra transceiver. Ensuring interoperability across the wider range of missions envisioned by the exploration program and Project Constellation requires a broader standard and more capable technology than has been thus far successfully demonstrated on Mars.

Efforts in partnership between Goddard Space Flight Center, the Jet Propulsion Laboratory and the Glenn Research Center are underway to develop a common standard for a Space Telecommunications Radio System (STRS) and advanced, interoperable software defined radios. STRS is an effort, supported by NASA Headquarters (Space Operations), that aims to establish the requirements and interoperability standards for a space capable software defined radio (SDR) architecture. By leveraging investment made in the DoD Joint Tactical Radio System (JTRS) program, STRS will provide to NASA a highly interoperable SDR architecture for use in manned and robotic spacecraft, rovers, and infrastructure assets.

As depicted in Figure 5, a Lunar

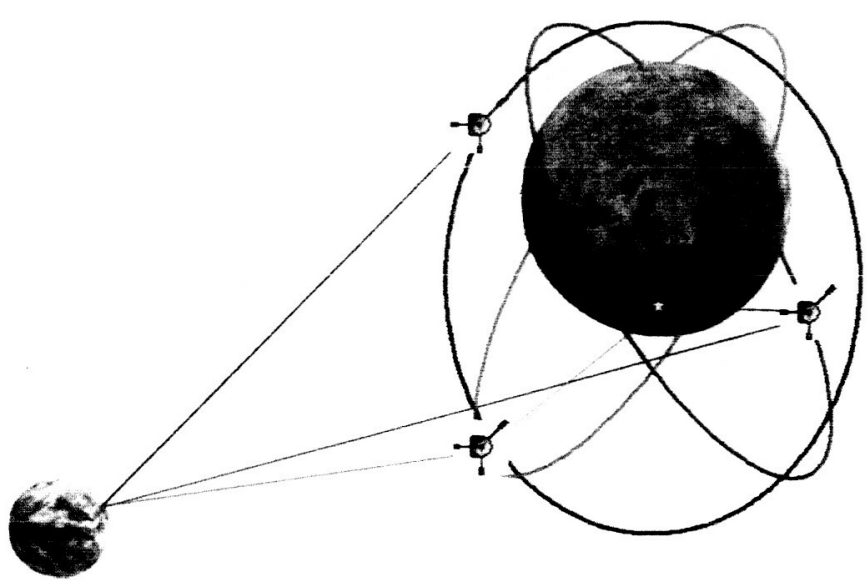

Figure 5 - Conceptual Lunar Communication Relay Network

communication relay network can provide continuous coverage of any point on the surface of the moon. Similar in architecture to the Mars Network, or the terrestrial Iridium constellation, the network would consist of a fleet of

5

American Institute of Aeronautics and Astronautics 
relay spacecraft in circular or elliptical orbits. Proper phasing of the orbits can ensure that at least two relay spacecraft are visible from any surface location at any given time. The spacecraft can provide services along the lines of the Earth based TDRSS, including communication and navigation support, providing a seamless operations concept between near-Earth and Lunar space.

In addition to basic communication and navigation services, advances in networking protocols and standards make possible the deployment of "Interuet-like" architectures in space. Relay communication systems envisioned by Goddard and JPL include IP and CCSDS packet routing, store-and-forward capabilities, and autonomous link detection and negotiation. By enabling relay providers to manage their own links through ad-hoc negotiations, increased network availability and operational flexibility is achieved. These capabilities may prove very useful, especially in deployed sensor web concepts in which many small sensors are scattered over the surface and are responsible for their own self-organization and communication.

With points of interest on the Moon located out of view of Earth, it is also desirable to reduce scheduling and network coordination between relay assets, user spacecraft, and mission planners and flight controllers. By permitting the network to be self-organizing in an ad-hoc manner, similar to terrestrial 802.11 wireless networking, communication can be accomplished with a minimum of Earth-based operator intervention.

The idea of an ad-hoc communication relay network is being investigated as a key element of the early Lunar Network in support of initial robotic exploration missions. Under this model, each robotic orbiter would carry with it a communication relay payload, and would become a network asset upon completion of its primary science or exploration mission. Missions originally placed into an appropriate orbit may assume their secondary relay role immediately. This is analogous to the Science Mission Directorate approach on Mars, and it would be a logical extension of the infrastructure supporting robotic exploration as it can expand with time to serve eventual human missions as well.

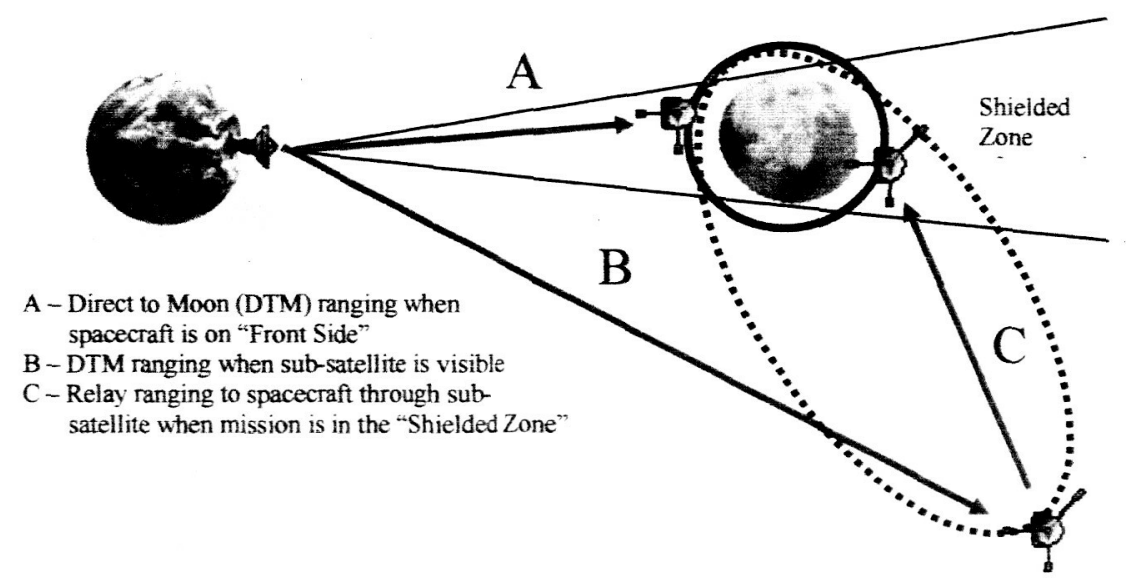

Figure 6 - Lunar Far Side Relay Concept

\section{Implementation of Lunar Proximity Network}

Both economic and physical factors demand that NASA accomplish as much as possible, and at the lowest possible mass for each mission. Economic in that the cost of sending mass to the Moon is significant (and nearly half of the mass sent to the Moon on any given mission is fuel). Physical in that the tidally locked rotation, and three-body gravity environment of cis-lunar space impacts both landed and orbital missions: landed missions to the far-side will never have direct views of Earth, while orbiting missions must make frequent orbit maintenance burns to maintain altitude. A series of low maintenance, "frozen" orbits have been identified by Goddard and JPL into which a spacecraft can be placed for its extended mission as a relay.

For missions not in low maintenance orbits, such as LRO's $50 \mathrm{~km}$ science orbit, frequent burns will be the norm. Each kilogram of spacecraft dry mass requires nearly a kilogram of fuel for a one year mission in a low lunar orbit. With mass (and fuel) at a premium, and an established operational need for relay communication in order to explore the far-side and many polar regions (as depicted in Figure 6), the argument for sharing resources through mission communication and navigation cross-support is strengthened.

6

American Institute of Aeronautics and Astronautics 
Each mission to the far-side (or into a location out of view of Earth such as a polar crater) requires relay communication to be successful. To ensure success, it is advisable to provide redundancy in communication assets. On a single spacecraft mission, this would ordinarily be achieved by providing a secondary communication payload on the host relay spacecraft. This is in part the reliability approach taken with the Electra Proximity Payload on the Mars Telecommunications Orbiter spacecraft. The probability of failure in both radios is unlikely, so the likelihood of a functional relay capability is high. The possibility does exist, however, for a spacecraft failure exclusive of the communication system to disable both radios. In the lunar problem, however, the extra mass of the (hopefully) unused communication system comes at a premium in terms of fuel which translates to cost.

If, instead of each mission providing its own redundancy and absorbing the associated cost, the mission is considered to be one of a series of mutually supportive missions. In this case, the entire program is treated as an integrated whole from the standpoint of communication and navigation infrastructure, and a new option becomes available. It is now possible to provide redundancy to ensure mission success by providing for redundant assets rather than redundant components. That is, with the likelihood of failure of a radio being nearly the same between two spacecraft, the likelihood that at least one of the two radios will be functional is increased in comparison to that of a traditional dual string redundant mission. This argument emerges from the fact that the second radio, on a second independent spacecraft, is immune to a failure (not necessarily in the radio) on the first spacecraft.

In addition to the increased redundancy for less mass that is achieved by employing a relay concept, the missions enjoy increased operational flexibility. The more spacecraft that are capable of relay support, the more available communication contacts will occur. This allows mission planners to select more freely from the available contacts, or to use greater amounts of bandwidth to return increasing science measurement volumes to Earth, again increasing the benefit of a mission to science and exploration.

It is possible to deploy proximity relay communication systems in several forms:

1. A proximity relay communication system can be created by assembling appropriate off-the-shelf transmitters, receivers and digital interfaces. These components are combined to provide an independent RF communication subsystem with its own interface to the spacecraft command and data handling system. This comes at the expense of increased component count, generally greater masses, and greater loading on the flight software. It can, however, be the least expensive option.

2. Relay optimized transceivers such as the JPL Electra are designed to be entire relay communication subsystems in one component. The Electra, and devices like it, combine a transmitter and receiver with a baseband processor to provide protocol and link management functions. The interface to the main spacecraft C\&DH system involves command and data transfers, with system maintenance being handled internally.

3. A third option, enabled by reconfigurable communication technologies is to integrate relay functions into the primary spacecraft communication system. In this case, one communication system serves both the function of TT\&C as well as relay. This option can provide the highest level of integration, but introduces additional complexity into the system.

A final factor in establishing a proximity network is the need for interoperable modulation, frequency, channel coding, framing, and protocols. A key to the success of the Mars Network concept is the development and use of the CCSDS Proximity-1 protocol, which calls for standard physical (frequency, modulation), link (coding, framing) interfaces, and basic network functionality in the form of reliable packet delivery. Over the Proximity-1 link, spacecraft can then exchange data using reliable transfer protocols, such as CCSDS CFDP ${ }^{7}$, which allow assured delivery of large files.

\section{Conclusion}

Unique factors affect the design of lunar exploration missions. In order to achieve science and exploration objectives, it is necessary to deliver probes and other mission elements to areas of the Moon that are permanently cut off from communication with Earth. Transmission of commands to and retrieval of data from these missions require proximity relay communication cross-support capability in another asset.

To reduce the overall program cost, and increase each mission's reliability, it is advisable for each long-duration orbiter to carry a proximity relay capable communication system. Missions should rely on each other for support and redundancy rather than requiring specific missions to carry high reliability, high cost, redundant systems to ensure mission success in the event of a failure. While requiring each long-term orbiter to provide single string relay support increases each mission's costs, total costs to the program are decreased, while program reliability is increased, as is operational flexibility and science return. 


\section{Acknowledgments}

The work described in this paper was carried out at NASA's Goddard Space Flight Center and at the Jet Propulsion Laboratory, California Institute of Technology under contract to NASA.

\section{References}

'The Vision for Space Exploration, National Aeronautics and Space Administration, NF-2004-01-334-HQ.

${ }^{2 " C l e m e n t i n e ~ M i s s i o n ~ I n f o r m a t i o n ", ~ N a t i o n a l ~ S p a c e ~ S c i e n c e ~ D a t a ~ C e n t e r, ~ G o d d a r d ~ S p a c e ~ F l i g h t ~ C e n t e r, ~}$ http://nssdc.gsfc.nasa.gov/planetary/clementine.html.

"Lunar Prospector Information", National Space Science Data Center, Goddard Space Flight Center, http://nssdc.gsfc.nasa.gov/planetary/lunarprosp.html.

${ }^{4}$ W.C. Feldman, et. al., "Fluxes of Fast and Epithermal Neutrons from Lunar Prospector: Evidence for Water Ice at the Lunar Poles," Science, Vol. 281, Issue 5382, 4 September 1998, pp 1496-1500.

${ }^{5}$ Proximity-1 Space Link Protocol, current version, Consultative Committee on Space Data Systems, CCSDS 211.0-B-1.

${ }^{6}$ Edwards, C., et. al., "The Electra Proximity Link Payload for Mars Relay," 54th International Astronautical Congress, IAF03-Q.3.a.06, Bremen, Germany, October 2003.

${ }^{7}$ CCSDS File Delivery Protocol, current version, Consultative Committee on Space Data Systems, CCSDS 727.0-B-2. 